\title{
A Lightweight Approach to Simulate a 2D Radar Coverage for Virtual Maritime Environments
}

\author{
*Warunika Ranaweera ${ }^{\dagger}$, Shazan Jabbar ${ }^{\dagger}$, Ruwan Wickramarachchi ${ }^{\dagger}$, Maheshya Weerasinghe ${ }^{\dagger}$, Naduni Gunathilake ${ }^{\dagger}$, \\ Chamath Keppitiyagama ${ }^{\dagger}$, Damitha Sandaruwan ${ }^{\dagger}$ and Prabath Samarasinghe ${ }^{\dagger}$ \\ $\dagger$ University of Colombo School of Computing, Sri Lanka
}

\begin{abstract}
Virtual simulation systems present a cost effective way of training the mariners to navigate a ship in a realistic maritime environment. To offer a better training session, a need arises to model other tools and components as a part of the virtual simulation system, such as radar, sonar and telescope, that are used to navigate a vessel in the real world. Taking a light-weighted approach, we developed a virtual radar coverage for the Vidusayura virtual maritime learning environment. It simulates an actual marine radar, which gathers information of its surroundings from the virtual environment with which a trainee naval officer interacts. The impact and the effectiveness of the virtual radar system, in terms of the trainee, is also analyzed against a real radar simulation system.
\end{abstract}

Index Terms-Radar, virtual reality, maritime simulation

\section{INTRODUCTION}

With the advancement of technology, simulation and virtual reality related techniques have become the state of the art methodologies in naval and maritime training. Virtually simulated maritime environments providing a safer, cost effective and an engaging training experience to the trainees, has become more effective than what was accomplished by traditional training sessions over the past few decades. Users are able to interact with virtual maritime environments in real time, getting appropriate responses back from the environment itself; a process which resembles a real life marine environment.

Nautical chart plotters which use artificially generated GPS, radar and AIS data, and stand alone radar and sonar simulators [2] have improved the productivity of training sessions even before virtual maritime environments became popularly in use. Even though the aforementioned techniques improved the productivity of traditional training sessions, the significance of them as standalone components diminished with the origination of the virtual maritime environments.

In the process of providing a rich simulation system to naval and maritime training, the necessity of modelling equipments and control panels, such as telescopes [3], radar and sonar, and integrating these to work with the virtual maritime environments in real time has become vital. Virtual maritime environment along with these supportive equipments and panels create a mixed reality, where the user experience would almost be similar to the physical maritime environment training experience.
Our work intends to provide a better training experience by modelling a 2D radar coverage for the Vidusayura virtual maritime environment [4]. Although standalone radar simulation systems are optimised to improve the radar reading skills of trainees, it lacks in providing a real-time data feed from an environment the user currently interacts with. Thus the users miss the opportunity to gain an experience by operating a radar while navigating a real ship in a maritime setting. We propose our 2D virtual radar model as a solution to eliminate the aforementioned disadvantage by allowing the radar coverage to work with the virtual environment in real time. The Vidusayura virtual maritime environment was configured to output the ship and terrain related information into the virtual radar system, which, upon receiving the information, visualized a 2D radar coverage resembling a real radar system.

This paper presents our light-weighted approach taken to design and develop the virtual radar system, its integration with the Vidusayura ship simulator, as well as the effectiveness of such a system with respect to a trainee who navigates a ship in the virtual maritime environment. In the forthcoming section, the importance of a virtual marine radar is discussed further in detail. Section 3 is entirely dedicated to introduce the light-weighted approach we have taken when designing and implementing the virtual radar and to describe how it tallies with the concept of a real world radar. A discussion is carried out in the succeeding section presenting the results of the experiment, and its accuracy, while section 5 concludes and presents the future improvements to be carried out based on our work as a whole.

\section{RADAR Simulations In MARITIME ViRTUAL ENVIRONMENTS}

The marine radar is a device which helps to determine the obstacles around a ship, moving or stationary, and their approximate distance from the ship itself. A radar is most readily useful when the ship is nearing a shore, during which the sea levels start varying and numerous obstacles begin to appear without notice. The radar display shows an abstract circular map of the whole area with a pre-specified radius, centering the ship. Modern radar systems are equipped with several components to get the desired details of the surroundings of a ship, such as the radius of the displaying area. 
During a practical scenario, understanding a marine radar and extracting the crucial information which helps the decision making process, requires experience and skill; hence a proper training. Thus providing a first hand experience of a marine radar is vital during the training process offered to a mariner. Stand alone radar simulators [2], [5] are in use today to provide this required training. Such radar simulators deliver pseudoraw signals to imitate a real world radar [6], and the trainee is presented with a radar display that demonstrates the information collected from the simulated environment. Since the trainee is not interacting with that particular environment, the information presented by the radar would only improve the trainee's understanding of the typical radar readings. Hence, the fundamental intention of deploying the radar to provide help in maneuvering a ship is not fulfilled. The trainee should experience the guidance provided by a radar to direct a ship safely through the obstacles.

Virtually simulated maritime environments are the most widely used low-cost training technique for ship navigation. The Vidusayura ship simulation system, which allows six degrees of freedom ship motions with respect to rudder, throttle and environmental conditions [7], is one such virtual maritime learning and training environment. When a trainee navigates his ship, he interacts with the virtual environment in real time. If the aforementioned technique of radar simulation is to be integrated with the virtual environment, which the trainee interacts with while he navigates the ship, it should provide more effective end results than occupying a standalone radar system with an unreal set of information. As our experiment, we developed a virtual radar simulator for the Vidusayura maritime virtual environment, which gathers information of the surrounding area from the virtual environment itself, and displays them in real time according to the position of the virtual ship which is navigated by a trainee mariner.

When a trainee starts navigating a ship inside Vidusayura, the virtual radar system gathers the current ship position and the information on other ships that are in the surrounding area, either moving or stationary, from the server side of Vidusayura. It uses a light-weighted approach to gather the geographical information of the terrain, which involves a pixel matrix known as a height map. The approach taken to develop the conceptual model of the virtual radar is discussed in detailed in the succeeding section. By integrating Vidusayura ship simulator and the virtual radar system, we intend to provide both the experiences of operating a working radar, and using the critical radar readings properly to navigate the ship safely in a realistic maritime environment.

\section{System DESIGN OF A REAL-TIME ViRTUAL RADAR SIMULATION}

As a part of our work, we designed and implemented a virtual radar simulation system, which takes a light weight approach to represent the abstract details of the virtual environment.

\section{A. System Design}

The concept of the radar, upon which a real marine radar is built, was studied in order to initiate the designing phase of the virtual radar system. It was later mapped to a virtual world to achieve the lightweight software approach to build the concept of the 2D radar coverage for Vidusayura virtual maritime environment.

1) Concept of a real marine radar: Real marine radars emit electromagnetic energy pulses (i.e. radio waves) to detect the geographical setting, obstacles and other ships in its coverage [8]. The energy pulse is reflected back to the radar device by the objects in its path, which will then be used to find the approximate positions of the surrounding objects [9].

"The radar display is often referred to as the plan position indicator (PPI). On a PPI, the sweep appears as a radial line, centered at the center of the scope and rotating in synchronization with the antenna. Any returned echo causes a brightening of the display screen at the bearing and range of the object." [10].

2) Concept of the virtual radar: The virtual radar introduces the concept of gathering geographical information from a heightmap of a virtual environment to simulate a radar system. A heightmap is a $2 \mathrm{D}$ interpretation of a $3 \mathrm{D}$ terrain which can be envisioned as a grayscale image, with black representing the minimum height and white representing the maximum height [11].
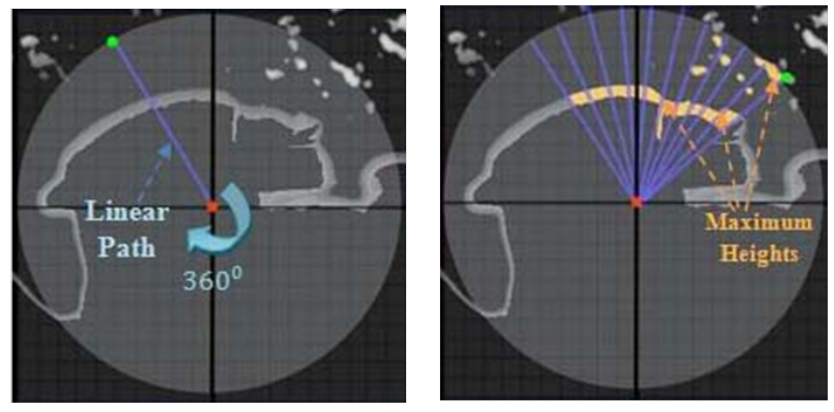

Fig. 1: Using the navigation ship coordinates as the origin

The virtual radar uses an algorithmic method to read the bitmap of a heightmap image and uses the intensity of its pixels as height values of a terrain. The logical next step in our concept is to calculate the maximum heights, analogous to pixel values laid on a linear pathway. As shown in Figure 1 we use the navigational ship coordinates as the origin of the radar, together with other coordinates of a precise radar target within a user-specified range inside the terrain to define the linear pathway.

In this concept radar images are generally a map view of maximum heights for a specified area adjoining the searched radar space. Depending on the intensity of the precipitation, different colors will appear on the map. Each color on the radar display will correspond to a different level of height.

3) Mapping the concept to a virtual world: Although the basic concept behind marine radar is simple, the theory has 
to be mapped carefully when it is applied to a virtual, physically intangible, simulation environment. The electromagnetic waves transmitted by a real radar are reflected only if they meet an electrically leading surface, which situationally becomes the maximum height along that particular line of sight. The reflected waves signal the radar that an obstacle lies in its direction of propagation. It is slightly similar to the concept of the virtual radar.

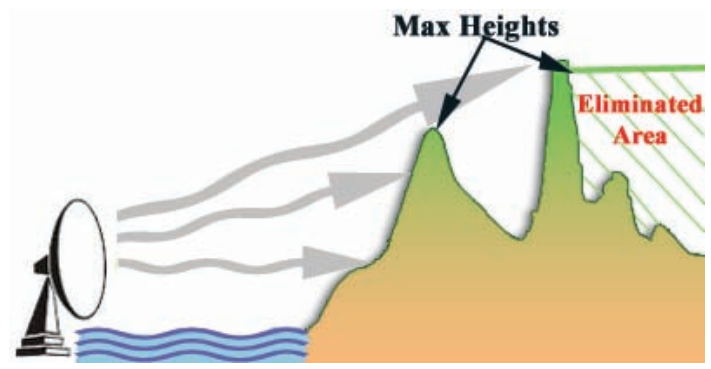

Fig. 2: Maximum-height detection of a real radar

Since the height map represents the geographical height variation of the terrain upon which the virtual world is built, it can be used to identify the height variation from the pixel values on a straight line (eg. radius). Thus the search of the maximum height based on the pixel values along a straight line will eventually provide the "peaks and valleys" until it meets the pixel that corresponds to the maximum height. As per the concept of wave propagation in real radar, the area beyond the maximum height is not visible since last point the propagated wave is returned back is at the maximum height. When it comes to the virtual radar, the area beyond the maximum height can be represented by setting the corresponding pixels to the value of the pixel that holds the maximum height of the line as depicted in Figure 2. This approach of exploiting the advantage of a height map can be considered light weighted since it does not require modelling the complex wave propagation and detection methodologies inside a virtual environment.

\section{B. System Implementation}

The system implementation of the virtual radar system consists of two components; a radar sever to generate the radar image view as an MJPEG video stream, as well as a client program to display the video stream with controls. The radar server has several key functionalities which will be discussed under this section.

1) Pre-processing the height map image: Since a height map represents the height variation of the terrain from the color values of a grayscale image, it is an initial need to extract the pixel values of the height map image. Thus a matrix of pixel values ("Pixel Matrix") is obtained by passing the height map image through the procedures of an image library.

2) Retrieval of the real time ship and environmental updates: Since Vidusayura is used as the maritime simulation system to incorporate the virtual radar system, it is needed to be in compliance with the real time changes of the maritime environment. The server program of Vidusayura calculates the ship positions and other environmental changes by using motion prediction algorithms [4]. Calculated positions and the real time changes of the environment can be obtained as a raw data stream by multiple clients via connecting to the Vidusayura server. The virtual radar system, being a client of the Vidusayura server, obtains the predicted positions of the objects in the environment which are intended to display on the updated radar view. Since the radar system is implemented to simulate the navigational radar of a ship, movements of the "own ship" should have to be taken in to consideration. Thus the origin of the radar is tied to the position of the ship which is navigated by the trainee.

3) Maximum height detection using a circular search: In a real radar, upon the process of a "sweep", the view gets updated from the detected objects along the straight line the radar wave has propagated at that particular moment. To simulate the "sweep" effect and to display updates in the environment, a circular search of objects from the center of the radar view is used. For the circular search of the "Pixel Matrix", the Bresenham's line drawing algorithm [12], [13] is used to get the pixel values by locating the pixels which lie along the radius from a particular angle. Then the algorithm to detect maximum heights is used to scale the pixel values which vary from 0 to 255 to height values of the terrain by considering the sea level is 0 . Values of the pixels that falls in the area beyond the maximum height is set to the pixel value of the maximum height itself.

4) Populating the radar image: Soon after detecting the peaks and valleys and the maximum heights, along with the area to be eliminated, the modified pixel values along the radius are inserted in to a new matrix ("Display Matrix") which is maintained to populate the updated radar image from the pixels. When the ship moves, the origin of the radar should tied to the ship's position, hence changing the radar view according to the moving ship. The movement of the point of origin is eliminated by mapping the ship coordinates back to the coordinates of the point of origin, while changing the coordinates of all the surrounding pixels of the Display Matrix relative to the modified ship coordinates, as illustrated in Figure 3.

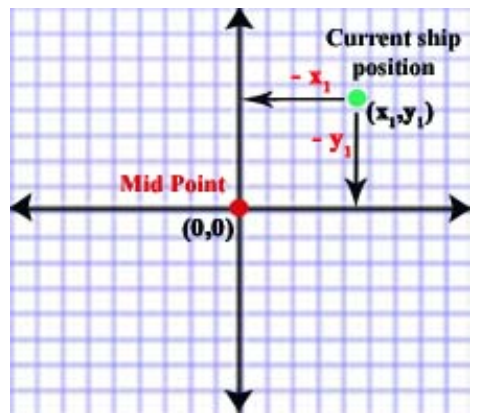

Fig. 3: Mapping the moving ship coordinates to the point of origin

Display Matrix is now passed through the Bresenham's Line 
Drawing Algorithm again to save the updated radar view as an image file.

5) Streaming the radar view to Clients: The last functionality of the Radar server is to generate the video stream of the radar view that can be viewed by multiple clients. Multipart HTTP headers are used to generate the MJPEG video stream from the images saved continuously over the time. Since the video stream which is broadcasted from the server uses HTTP headers it can be displayed by the Radar Client program, which is consisting of a MJPEG viewer or a web browser.

\section{Discussion And ANALYsis}

As elaborated in the prior section, our work provides a lightweight approach to simulate the real world 2D radar effect. The proposed virtual radar system is integrated with Vidusayura to collaboratively work in real time with the maritime virtual environment. In real radar scenarios, electromagnetic waves allow determining the distance between the reflective objects and the originated ship by measuring the running time of the transmitted pulses. With the use of a scaled height map, determining the distances to the reflective objects is also possible in the proposed virtual radar system. To ensure the effectiveness of our work several test runs of the virtual radar coverage, integrated with Vidusayura ship simulator, were analysed, and compared against a real radar of a real ship in the physical environment.

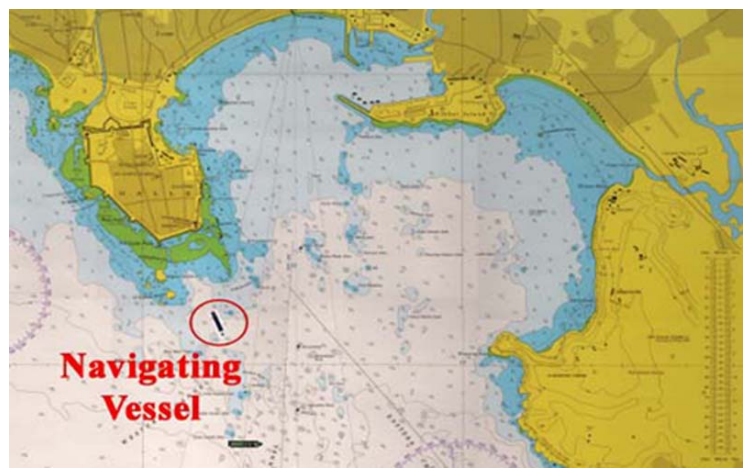

Fig. 4: The Electronic Chart Display and Information System of Vidusayura, displaying the geographical location of the vessel (Galle Harbour, Sri Lanka)

At a given moment, a single point of the test run is paused and analyzed. Three distinct views from the three components, the virtual radar, 2D map of the real environment and the Vidusayura virtual environment, are taken during that particular moment of pause and compared against each other.

In this test run the Vidusayura ship simulator demonstrates a vessel navigation in the maritime environment around the Galle harbour of Sri Lanka. Figure 4 represents the navigating vessel's geographical location in the simulated Electronic Chart Display and Information System (ECDIS) of Vidusayura. The radar coverage of that vessel, simulated by our radar system, is shown in Figure 5. The Figure 6 displays the 3D visual representation of the navigating vessel in Vidusayura. Since the aforementioned 3 components (the virtual radar, ECDIS and Vidusayura) are collaborated with each other, three distinct views of the integrated system are well synchronized during a real time training session. We

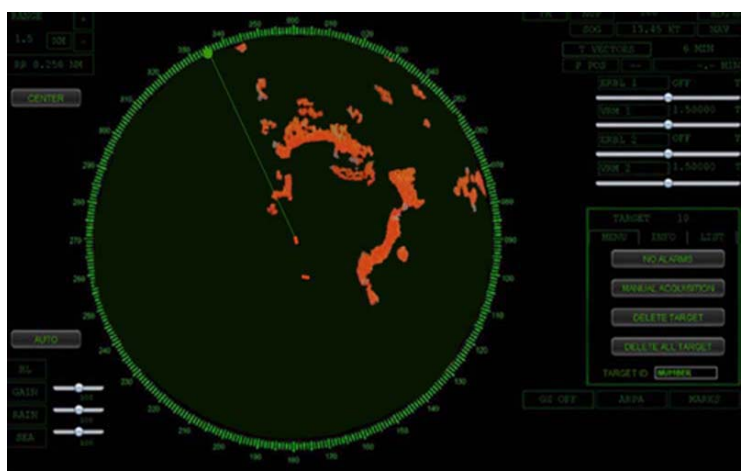

Fig. 5: Produced radar coverage of the navigating vessel

tested the effectiveness of the complete system when trainees interactively navigate the vessels in the virtual environment while obtaining information from the virtual radar.

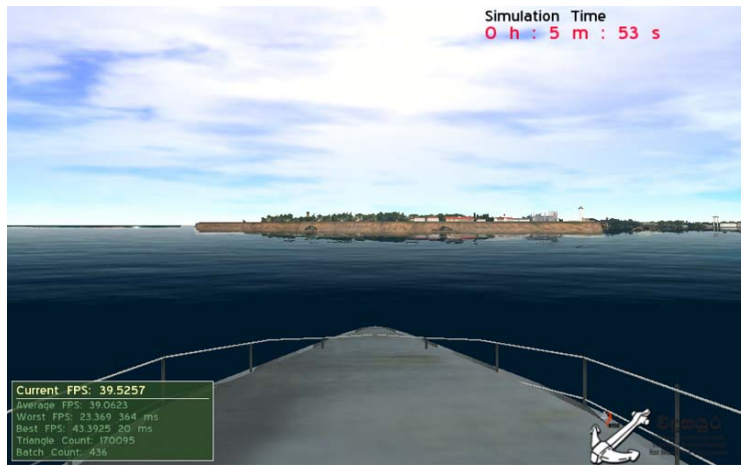

Fig. 6: 3D visual representation of the navigating vessel in Vidusayura

\section{CONClusions And Future Work}

The simulation of the real world radar equipments for training purposes targets on improving the radar operating skills of a trainee mariner. Although stand alone radar simulators are widely in use today, they fail to provide the necessary training required for improving the ship navigation skills of a trainee mariner with the use of a marine radar. Of particular concern, this paper recognizes the need to integrate the radar simulators with virtual maritime environments, operating from the information acquired from it in real time.

We present the currently designed and developed virtual 2D radar converge, which is integrated with the Vidusayura ship simulation system in order to enhance the training process provided to the trainees who navigate the vessels in the virtual maritime setting. The design of the virtual radar system follows a lightweight approach, during which the advanced concepts of the real world radar is simplified to be feasible 
enough for a virtual maritime environment. The end results presented in the discussion proves the efficiency and the accuracy of the lightweight approach taken to model a $2 \mathrm{D}$ radar converge for virtual environments.

Although, our virtual radar simulator solves the basic operations of a real radar system, some future modifications should be applied to the system to further enhance the functionalities while providing a more accurate radar coverage. The atmosphere, different weather conditions and material of the reflecting object directly affect the energy transmitted by the signal as well as the radar coverage. Our system should be further enhanced to simulate such different conditions and their influences on the radar output. And also, the system should be enhanced to vary the range of the radar coverage according to the user perception. The sonar which is used to measure the depth level of the sea, can also include into this radar system to get better measurements to navigate the ship in the sea without fearing the obstacles.

\section{ACKNOWLEDGEMENTS}

We would like to acknowledge the Sri Lanka Navy for their support provided during the initial development and testing phases of the virtual marine radar system.

\section{REFERENCES}

[1] J. Dean and S. Ghemawat, "Mapreduce: simplified data processing on large clusters," Communications of the ACM, vol. 51, no. 1, pp. 107-113, 2008.

[2] X. Tong, J. Pang, and Y. Li, "Maritime police warship radar simulation training system," in Electrical Electronics Engineering (EEESYM), 2012 IEEE Symposium on, june 2012, pp. 666-669.
[3] S. Jabbar, R. Wickramarachchi, W. Ranaweera, A. Weerasinghe, N. Gunathilake, C. Keppitiyagama, D. Sandaruwan, and P. Samarasinghe, "Virtual eye: A sensor based mobile viewer to aid collaborative decision making in virtual environments," in Advances in ICT for Emerging Regions (ICTer), 2012 International Conference on, 2012.

[4] D. Sandaruwan, N. Kodikara, R. Rosa, and C. Keppitiyagama, "Realtime ship motion prediction system," Proceedings of the Computer Games Multimedia and Allied Technology 09 (CGAT 09), vol. 6, 2009.

[5] A. Arnold-Bos, A. Khenchaf, and A. Martin, "Bistatic Radar Imaging of the Marine Environment-Part II: Simulation and Results Analysis," Geoscience and Remote Sensing, IEEE Transactions on, vol. 45, no. 11 pp. 3384-3396, nov. 2007.

[6] A. Arnold-Bos, A. Khenchaf, and Martin, "Bistatic Radar Imaging of the Marine Environment-Part I: Theoretical Background," Geoscience and Remote Sensing, IEEE Transactions on, vol. 45, no. 11, pp. 3372-3383, nov. 2007.

[7] D. Sandaruwan, N. Kodikara, C. Keppitiyagama, R. Rosa, M. Jayawardena, and P. Samarasinghe, "User perception of the physical-behavioral realism of a maritime virtual reality environment," in Computer Modelling and Simulation (UKSim), 2012 UKSim 14th International Conference on, march 2012, pp. 172-178.

[8] A. G. B. of Meteorology. (2012) How Radar Works. [Online]. Available: http://www.bom.gov.au/australia/radar/about/what_is_radar.shtml

[9] L. Varshney, "Radar Principles," Syracuse Research Corporation, NY, Tech. Rep., oct. 2002.

[10] P. Griffes, Atlantic Boating Almanacs Sandy Hook, Nj To St. John River, $\mathrm{Fl} \&$ Bermuda. ProStar Publications.

[11] R. M. Smelik, K. J. D. Kraker, S. A. Groenewegen, T. Tutenel, and R. Bidarra, "A Survey of Procedural Methods for Terrain Modelling," in Proceedings of the CASA Workshop on 3D Advanced Media In Gaming And Simulation (3AMIGAS), 2009.

[12] J. Foley, Computer graphics: principles and practice. Addison-Wesley Professional, 1996.

[13] J. E. Bresenham, "Algorithm for computer control of a digital plotter," IBM Systems Journal, vol. 4, no. 1, pp. 25 -30, 1965. 